\title{
Estrutura e composição florística da vegetação lenhosa em cerrado rupestre na transição Cerrado-Floresta Amazônica, Mato Grosso, Brasil
}

\author{
Leandro Maracahipes ${ }^{1,3}$, Eddie Lenza ${ }^{1}$, Beatriz Schwantes Marimon ${ }^{1}$, Edmar Almeida de Oliveira ${ }^{1}$, \\ José Roberto Rodrigues Pinto ${ }^{2}$ \& Ben Hur Marimon Junior ${ }^{1}$ \\ ${ }^{1}$ Programa de Pós-graduação em Ecologia e Conservação, Universidade do Estado de Mato Grosso - UNEMAT, \\ Campus de Nova Xavantina, CP 08, CEP 78690-000, Nova Xavantina, MT, Brasil \\ ${ }^{2}$ Programa de Pós-graduação em Ciências Florestais, Universidade de Brasília - UnB, \\ CEP 70910-900, Brasília, DF, Brasil \\ ${ }^{3}$ Autor para correspondência: Leandro Maracahipes, e-mail: Imaracahipes@gmail.com
}

MARACAHIPES, L., LENZA, E., MARIMON, B.S., OLIVEIRA, E.A., PINTO, J.R.R. \& MARIMON JR., B.H. Structure and floristic composition of woody vegetation in cerrado rupestre in the Cerrado-Amazonian Forest transition zone, Mato Grosso, Brazil. Biota Neotrop. 11(1): http://www.biotaneotropica.org.br/v11n1/ en/abstract?article+bn02111012011.

\begin{abstract}
This study aimed to analyze the floristic composition and the structure of a savanna on rocky soil ("cerrado rupestre") woody vegetation (including monocots and lianas) in the Cerrado-Amazon Forest transition zone located at Parque Municipal do Bacaba, Nova Xavantina, State of Mato Grosso (14 $41^{\prime}$ S and 52 $20^{\circ}$ W), and compare it with other cerrado stricto sensu studies. Ten $20 \times 50 \mathrm{~m}$ plots were randomly established, within which all live and dead woody plants with at least $3 \mathrm{~cm}$ of trunk diameter at $30 \mathrm{~cm}$ above ground level $\left(\mathrm{DSH}_{30} \geq 3 \mathrm{~cm}\right)$ were measured. The cerrado rupestre showed high density (3,766 live individuals), richness ( 85 species, 67 genera and 34 families) and basal area $\left(15.72 \mathrm{~m}^{2} \mathrm{ha}^{-1}\right)$, as well as high levels of Shannon-Wiener species diversity $\left(\mathrm{H}^{\prime}=3.47\right)$ and evenness $(\mathrm{J}=0.78)$ indices. The most important species were Erythroxylum suberosum, Qualea parviflora, Anacardium occidentale, Kielmeyera rubriflora and Vatairea macrocarpa. The greatest floristic similarity found between typical cerrado and "cerrado rupestre" communities from lower altitude areas of Northeastern Mato Grosso suggest that, in this region, altitude and geographical distance influence the species composition, regardless of the substrate. The community presented unimodality in the heights distribution and is mostly compounded by shrubby individuals with height $<3 \mathrm{~m}$ and DSH $<5 \mathrm{~cm}$. We suggest that floristic and phytosociological studies conducted in "cerrado rupestre" should adopt DSH $\geq 3 \mathrm{~cm}$ and include monocot and liana species, so as to more realistically represent the vegetation richness, species composition and structure. The high species richness and diversity registered in this study might be related to this "cerrado rupestre" pre-Amazonian location, which emphasizes the importance of "Parque do Bacaba" maintenance as a guarantee of its biological diversity protection.
\end{abstract}

Keywords: Cerrado-Amazon boundaries, phytosociology, Erythroxylum suberosum, savanna, Eastern Mato Grosso.

MARACAHIPES, L., LENZA, E., MARIMON, B.S., OLIVEIRA, E.A., PINTO, J.R.R. \& MARIMON JR., B.H. Estrutura e composição florística da vegetação lenhosa em cerrado rupestre na transição CerradoFloresta Amazônica, Mato Grosso, Brasil. Biota Neotrop. 11(1): http://www.biotaneotropica.org.br/v11n1/pt/ abstract?article+bn02111012011.

Resumo: O objetivo desse estudo foi determinar a composição florística e a estrutura da vegetação lenhosa (incluindo monocotiledôneas e lianas) em cerrado rupestre na zona de transição Cerrado-Floresta Amazônica, no Parque do Bacaba, em Nova Xavantina, MT (14 $41^{\circ}$ 'S e 52 $20^{\circ}$ W) e compará-las com outros estudos de cerrado sentido restrito. Foram demarcadas aleatoriamente 10 parcelas de $20 \times 50 \mathrm{~m}$, nas quais foram medidos os indivíduos vivos e mortos em pé com diâmetro mínimo a $30 \mathrm{~cm}$ do solo (DAS) $\geq 3 \mathrm{~cm}$. O cerrado rupestre apresentou alta densidade (3.766 indivíduos vivos), riqueza florística (85 espécies, 67 gêneros e 34 famílias) e área basal $\left(15,72 \mathrm{~m}^{2} \mathrm{ha}^{-1}\right)$, e ainda elevado valor do índice de diversidade de espécies de Shannon-Wiener $\left(H^{\prime}=3,47\right)$ e equabilidade de Pielou $(\mathrm{J}=0,78)$ em relação às áreas comparadas. As espécies com maior valor de importância foram Erythroxylum suberosum, Qualea parviflora, Anacardium occidentale, Kielmeyera rubriflora e Vatairea macrocarpa. A maior similaridade florística entre comunidades de cerrado típico e rupestre localizadas em áreas com menores altitudes do leste mato-grossense sugere que nessa região a proximidade geográfica e a altitude exercem influência sobre a composição de espécies, independentemente do substrato. A comunidade apresentou distribuição de alturas unimodal, predominando indivíduos de porte arbustivo com altura $<3$ m e DAS $<5 \mathrm{~cm}$. É sugerido aqui que estudos florísticos e fitossociológicos realizados em cerrado rupestre empreguem DAS mínimo de $3 \mathrm{~cm}$ e incluam espécies de monocotiledôneas e de lianas para representar de maneira mais realística a riqueza e composição de espécies e a estrutura da vegetação. A elevada riqueza e diversidade de espécies registrada no presente estudo podem estar relacionadas à posição pré-Amazônica deste cerrado rupestre, enfatizando a importância da manutenção do Parque do Bacaba no sentido de garantir a proteção de sua diversidade biológica. Palavras-chave: limites Cerrado-Amazônia, fitossociologia, Erythroxylum suberosum, savana, leste mato-grossense. 


\section{Introdução}

O patrimônio natural brasileiro apresenta grande importância para a conservação da biodiversidade global, devido à sua extensão territorial, à diversidade e ao endemismo de espécies, ao patrimônio genético e à heterogeneidade ecossistêmica de seus biomas (Assunção $\&$ Felfili 2004). Nesse sentido, o bioma Cerrado, a segunda maior formação vegetal brasileira em extensão, possui uma das mais ricas e diversas floras do mundo, com cerca de 11.627 espécies vasculares de plantas superiores nativas (Mendonça et al. 2008), além de ampla variação regional na composição florística (Ratter et al. 2003, Bridgewater et al. 2004) e de um elevado número de espécies endêmicas (Ratter et al. 1997, Machado et al. 2004).

Fisionomicamente, o Cerrado é caracterizado por vegetação tipicamente savânica, com menor ocorrência de formações florestais e campestres (Ribeiro \& Walter 2008). Entre as fitofisionomias savânicas, destaca-se o cerrado sentido restrito, que possui cobertura arbórea variando de 10 a $60 \%$ (Eiten 1979) e que foi subdividido por Ribeiro \& Walter (2008) em cerrado denso, cerrado típico, cerrado ralo e cerrado rupestre, com base nas densidades dos componentes arbustivo-arbóreo e subarbustivo-herbáceo e nas propriedades do substrato. De acordo com estes autores, o substrato do cerrado rupestre diferencia-o das demais fitofisionomias por ocorrer em solos rasos e com presença de afloramentos rochosos. Os solos do cerrado rupestre são classificados como Neossolo Litólicos, onde as plantas se fixam nas fendas entre as rochas, onde ocorrem acúmulo e decomposição de matéria orgânica e maior deposição de areia resultante do intemperismo das rochas (Reatto et al. 2008, Ribeiro \& Walter 2008).

O cerrado rupestre pode ocorrer em trechos contínuos, mas geralmente em mosaicos com outras formações vegetais (Ribeiro \& Walter 2008), apresenta flora com alto grau de endemismo (Alves \& Kolbek 2010), várias espécies ameaçadas de extinção (Mendonça \& Lins 2000) e ocorrência de espécies típicas de formações savânicas do bioma e algumas espécies restritas (Pinto et al. 2009, Lima et al. 2010). A diversidade de plantas vasculares dos cerrados rupestres é superior àquela encontrada na maioria das regiões do mundo (Amaral et al. 2006).

Os processos de ocupação e exploração do Cerrado em vários estados brasileiros deixaram a cobertura vegetal primitiva reduzida a pequenos remanescentes, comprometendo a integridade da fauna e flora presentes no bioma (Klink \& Machado 2005, Silva et al. 2006). Muitas vezes, as áreas de cerrado rupestre não são utilizadas pela agricultura intensiva, por serem normalmente de difícil acesso, pois estão associados a afloramentos rochosos (Ribeiro \& Walter 2008). Essas áreas podem, dessa forma, representar importantes refúgios para a flora e a fauna do Cerrado, frente ao acelerado processo de ocupação humana. A área do presente estudo localiza-se na transição entre os biomas Cerrado e Floresta Amazônica na região abrangida pelo "arco do desmatamento", que tem levado a uma redução drástica de sua área de cobertura vegetal original (Nogueira et al. 2008).

Neste sentido, considerando a necessidade de estudos e conservação da comunidade vegetal de cerrado rupestre (Martinelli 2007), os levantamentos florísticos e fitossociológicos são excelentes ferramentas para a determinação da riqueza e diversidade locais e regionais (Felfili \& Felfili 2001). Além disso, estudos dessa natureza, fornecem subsídios para posteriores estudos de dinâmica, recuperação de áreas degradadas e delimitação de unidades de conservação (Marimon et al. 1998, Felfili et al. 2002) bem como para estudos fitogeográficos (Ratter et al. 1997, Bridgewater et al. 2004, Silva et al. 2006), essenciais na determinação de estratégias de conservação. Desse modo, o presente estudo teve como objetivo analisar a composição florística e a estrutura fitossociológica da vegetação lenhosa em uma área de cerrado rupestre localizada na zona de transição entre os biomas Cerrado e Floresta Amazônica, no Parque do Bacaba em Nova Xavantina, MT, comparando com outras fitofisionomias de cerrado sentido restrito do Cerrado.

\section{Material e Métodos}

$\mathrm{O}$ estudo foi desenvolvido em uma área de cerrado rupestre situada no Parque Municipal do Bacaba (CRPB) (14 $41^{\circ}$ ' $\mathrm{S}$ e $52^{\circ} 20^{\prime}$ W) no município de Nova Xavantina, leste de Mato Grosso, na região de transição entre os biomas Cerrado e Floresta Amazônica. A região é caracterizada pelo domínio de fisionomias savânicas e florestais típicas do bioma Cerrado e ainda formações florestais préamazônicas (Marimon et al. 1998, Felfili et al. 2002, Marimon et al. 2006). Os solos da região foram caracterizados como Latossolo Vermelho-Amarelo Distrófico, de textura média, bem drenado e com relevo plano (Prodeagro 1995). O clima é do tipo Aw, de acordo com a classificação de Köppen (Camargo 1963), sendo o período seco de maio a setembro e o chuvoso de novembro a março. O Parque do Bacaba apresenta uma área de aproximadamente 500 ha, onde a vegetação predominante é de cerrado sentido restrito, apresentando contato com áreas de cerradão e floresta (Marimon Jr. \& Haridasan 2005). O cerrado rupestre ocupa cerca de $6 \%$ da área do Parque do Bacaba se desenvolvendo entre 340 e $400 \mathrm{~m}$ de altitude sobre cristas de serra estreitas e alongadas, de terreno acidentado e com intenso afloramento rochoso de quartzito (Abad \& Marimon 2008) ou Neossolo Litólico.

Foi adotado o método de parcelas permanentes (Felfili et al. 2005), sendo estabelecidas aleatoriamente 10 parcelas de $20 \times 50 \mathrm{~m}$ ( $1 \mathrm{ha}$ ), com distância mínima de $50 \mathrm{~m}$ entre si e alocadas perpendicularmente às encostas dos afloramentos para contemplar possíveis variações em função do gradiente topográfico. Foram amostrados (altura total e diâmetro) de todos os indivíduos lenhosos (incluindo lianas e monocotiledôneas) vivos e mortos em pé, com diâmetro a $30 \mathrm{~cm}$ do solo $\left(\mathrm{DAS}_{30 \mathrm{~cm}}\right) \geq 3 \mathrm{~cm}$. A altura total foi obtida por meio de uma régua telescópica graduada de 9,5 m. Nos casos em que os indivíduos, dentro do limite de inclusão adotado, apresentaram ramificações abaixo de $30 \mathrm{~cm}$, todos os ramos foram medidos e posteriormente foi calculado o diâmetro quadrático das ramificações, conforme sugerido por Scolforo (1993). A identificação das espécies foi realizada por meio de comparações com o acervo do Herbário NX, Campus da UNEMAT de Nova Xavantina, consulta às bibliografias especializadas e envio a especialistas. O material botânico reprodutivo coletado encontra-se incorporado ao acervo do Herbário NX. O sistema de classificação botânica adotado para as famílias foi o proposto por APG III (Angiosperm..., 2009).

A diversidade de espécies foi calculada a partir do índice de Shannon-Wiener (H') e para a uniformidade (equabilidade) utilizou-se o índice de Pielou (J') (Magurran 1988). Para calcular os parâmetros fitossociológicos sugeridos por Müeller-Dombois \& Ellenberg (1974) utilizou-se o programa Mata Nativa 2 (Cientec 2006) e as equações foram baseadas em Brower \& Zar (1977) e Ludwing \& Reynolds (1988). Foram analisadas as distribuições dos indivíduos da comunidade em classes de alturas e de diâmetros, utilizando a equação proposta por Spiegel (1976). A riqueza de espécies foi estimada pelo método de rarefação (Gotelli \& Colwell 2001), a partir do programa EstimateS 8.2 (Colwell 2008). Para estimar o número de espécies que poderia ter sido amostrado foi utilizado o estimador Jackknife 1 (Magurran 1988).

Foram selecionados cinco estudos realizados em cerrado típico sobre Latossolos e cinco em cerrado rupestre sobre Neossolos Litólicos para comparações florísticas (riqueza, diversidade e equabilidade) e estruturais (densidade e área basal) com o cerrado rupestre do Parque do Bacaba. A análise de agrupamento foi efetuada 
com base na média de grupo (UPGMA= Unweighted Pair-Groups Method using Arithmetic Averages) para analisar a similaridade florística. Neste caso, o processo de fusão ou iteração é baseado na distância média mínima entre indivíduos e grupos (Kent \& Coker 1992). A análise de agrupamento foi realizada a partir do software NTSYS v. 2.1 (Rohlf 2000). Foram selecionadas apenas estudos que adotaram o método de parcelas, totalizando 1 ha de área amostral.

Para as comparações descritas acima foram considerados apenas os indivíduos com diâmetro $\geq 5 \mathrm{~cm}$, uma vez que os estudos desenvolvidos em formações savânicas de cerrado geralmente utilizam esse critério de inclusão (Felfili et al. 2005). Para avaliar os efeitos da distância geográfica sobre a composição florística, foram medidas as distâncias, em linha reta, entre as áreas comparadas e o cerrado rupestre estudado, utilizando as coordenadas geográficas de cada área, as imagens de satélite e a ferramenta para medição de distância por meio do programa Google Earth (www.earth.com/ intl/pt-br).

\section{Resultados}

No cerrado rupestre estudado, considerando todos os indivíduos com DAP $\geq 3 \mathrm{~cm}$, foram registradas 85 espécies, 67 gêneros e 34 famílias (Tabela 1), com densidade de 3.766 indivíduos.ha $^{-1} \mathrm{e}$ área basal de $15,72 \mathrm{~m}^{2} \cdot \mathrm{ha}^{-1}$. O índice de diversidade de espécies de Shannon-Wiener ( $\left.H^{\prime}\right)$ foi de 3,47 e a equabilidade $(\mathrm{J})$ de 0,78 . Foram registrados 118 indivíduos mortos (3\% do total de indivíduos) que juntos somaram área basal de $0,73 \mathrm{~m}^{2} . \mathrm{ha}^{-1}$ (4,7\% da área basal total). Três espécies de monocotiledôneas (Syagrus flexuosa, S. comosa e Vellozia squamata) e duas de lianas lenhosas (Arrabidaea cinnamomea e Banisteriopsis pubipetala) contribuíram com 193 indivíduos. Juntas, essas cinco espécies representaram 5,1\% dos indivíduos, 4,9\% do valor de importância (VI) e 3,2\% da área basal da comunidade estudada (Tabela 1).

A curva de acumulação de espécies apresentou tendência à estabilização a partir da sexta parcela, na qual $83(97,6 \%)$ das 85 espécies amostradas na área já haviam sido registradas (Figura 1). O número de espécies registrado contemplou aproximadamente $87 \%$ do número estimado para comunidade (Jackknife $1=97,6$ ), sugerindo que o esforço amostral foi suficiente na determinação da composição florística local (riqueza alfa) da flora lenhosa do cerrado rupestre estudado.

As 10 espécies mais importantes em VI (11,8\% do total de espécies) foram registradas nas 10 parcelas amostradas, e representaram $59,1 \%$ dos indivíduos, $45,8 \%$ do VI total e $56,6 \%$ da área basal total da comunidade. Erythroxylum suberosum, Kielmeyera rubriflora, Pseudobombax longiflorum, Erythroxylum tortuosum e Eugenia aurata alcançaram posição de destaque no VI em função das elevadas densidades relativas, a despeito do porte arbustivo ou subarbustivo dessas espécies. Por outro lado, devido ao porte tipicamente arbóreo, Anacardium occidentale e Dipteryx alata se destacaram em função dos maiores valores de dominância. Qualea parviflora, Vatairea macrocarpa e Heteropterys byrsonimifolia se destacaram em função dos elevados valores relativos de densidade e área basal (Tabela 1). Foram amostradas 10 espécies consideradas raras, por apresentar apenas 1 ind.ha ${ }^{-1}(11,8 \%$ das espécies e $1 \%$ do VI total) (Tabela 1).

A estrutura vertical da comunidade lenhosa estudada foi caracterizada predominantemente por indivíduos com porte arbustivo (alturas ente 1 e $3 \mathrm{~m}$ ), com 2.686 indivíduos vivos (71,3\% do total), seguida por indivíduos de porte arbóreo (> $3 \mathrm{~m})$, com 870 indivíduos ( $23,1 \%$ do total) e subarbustivo $(<1 \mathrm{~m})$, com 210 indivíduos $(5,6 \%$ do total) (Figura 2a). Das 85 espécies registradas na área, 76 (89\%) apresentaram pelo menos um indivíduo com porte arbustivo. Em

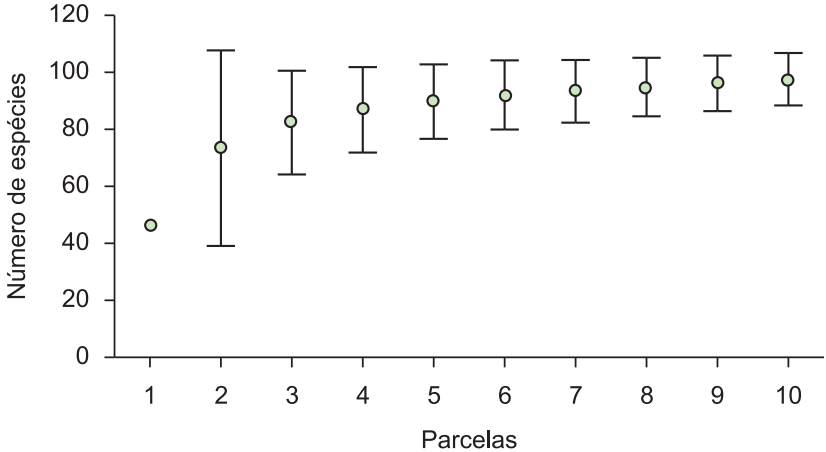

Figura 1. Curva de acumulação de espécies lenhosas $\left(\mathrm{DAS}_{30} \geq 3 \mathrm{~cm}\right)$ em um cerrado rupestre na transição Cerrado-Floresta Amazônica, porção Leste de Mato Grosso.

Figure 1. Rarefaction curve of a "cerrado rupestre" woody species ( $\mathrm{DSH}_{30}$ $\geq 3 \mathrm{~cm}$ ) in the Cerrado-Amazon Forest transition zone of Eastern Mato Grosso, Brazil.
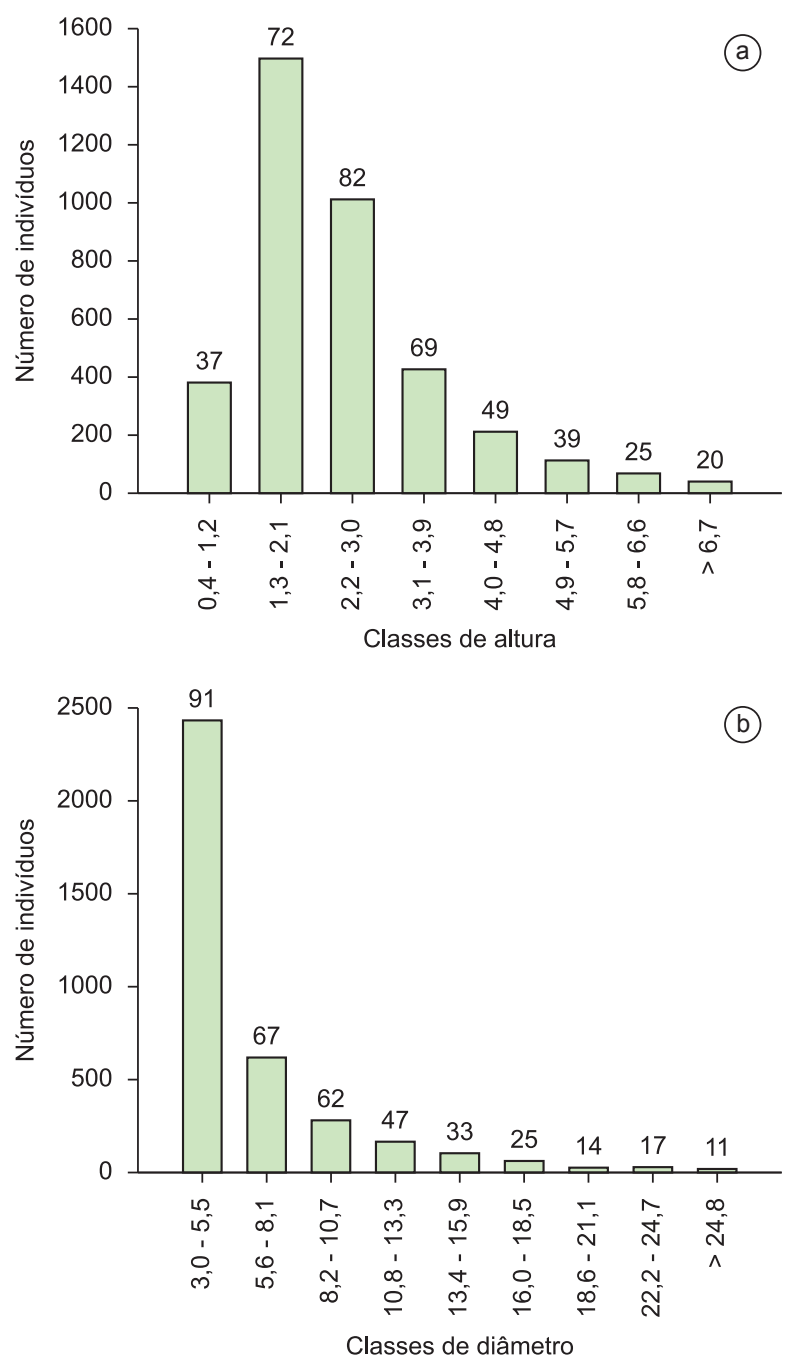

Figura 2. a) Distribuição das classes de alturas; e b) diâmetros dos indivíduos lenhosos vivos $\left(\mathrm{DAS}_{30} \geq 3 \mathrm{~cm}\right.$ ) do cerrado rupestre estudado. Os valores acima das barras indicam o número de espécies por classe.

Figure 2. a) Distribution of height; and b) diameter classes of the "cerrado rupestre" live woody individuals $\left(\mathrm{DSH}_{30} \geq 3 \mathrm{~cm}\right.$ ). The values above the bars indicate the number of species per class. 
Tabela 1. Parâmetros fitossociológicos das espécies lenhosas $\left(\mathrm{DAS}_{30} \geq 3 \mathrm{~cm}\right)$ do cerrado rupestre estudado. DA = Densidade absoluta; DR $=$ Densidade relativa; FA = Frequência absoluta; FR = Frequência relativa; DoA = Dominância Absoluta; DoR = Dominância Relativa e VI = Valor de Importância. Espécies em ordem decrescente de VI. Valores absolutos em hectare $\left(\mathrm{D}=\mathrm{N} \cdot \mathrm{ha}^{-1} \mathrm{e} \mathrm{Do}=\mathrm{m}^{2}\right.$.ha $\left.{ }^{-1}\right)$ e valores relativos em \%. * Liana; ** Monocotiledônea não lenhosa.

Table 1. Phytosociological parameters of the woody species $\left(\mathrm{DSH}_{30} \geq 3 \mathrm{~cm}\right)$ in the studied cerrado rupestre. $\mathrm{DA}=\mathrm{Absolute}$ density; DR = Relative density; FA = Absolute frequency; FR = Relative frequency $;$ DoA = Absolute dominance; DoR = Relative dominance and VI = Value of Importance. Species in decreasing order of VI. Absolute values in hectare $\left(\mathrm{D}=\mathrm{N} \cdot \mathrm{ha}^{-1}\right.$ and $\left.\mathrm{Do}=\mathrm{m}^{2} \cdot \mathrm{ha}^{-1}\right)$ and relative values in \%. * Liana; ** Non woody monocots.

\begin{tabular}{|c|c|c|c|c|c|c|c|c|}
\hline Espécie & Família & DA & DR & FA & FR & DoA & DoR & IVI \\
\hline Erythroxylum suberosum A. St.-Hil. & Erythroxylaceae & 539 & 14,31 & 100 & 2,17 & 1,01 & 6,45 & 22,94 \\
\hline Qualea parviflora Mart. & Vochysiaceae & 323 & 8,58 & 100 & 2,17 & 1,32 & 8,42 & 19,18 \\
\hline Anacardium occidentale $\mathrm{L}$. & Anacardiaceae & 99 & 2,63 & 100 & 2,17 & 2,05 & 13,02 & 17,83 \\
\hline Kielmeyera rubriflora A. St.-Hil. & Clusiaceae & 381 & 10,12 & 100 & 2,17 & 0,67 & 4,25 & 16,55 \\
\hline Vatairea macrocarpa (Benth.) Ducke & Fabaceae & 243 & 6,45 & 100 & 2,17 & 1,18 & 7,51 & 16,14 \\
\hline Heteropterys byrsonimifolia A. Juss. & Malpighiaceae & 176 & 4,67 & 100 & 2,17 & 0,68 & 4,29 & 11,15 \\
\hline Dipteryx alata Vogel & Fabaceae & 49 & 1,30 & 100 & 2,17 & 1,02 & 6,51 & 9,99 \\
\hline $\begin{array}{l}\text { Pseudobombax longiflorum (Mart. \& Zucc.) A. } \\
\text { Robyns }\end{array}$ & Malvaceae & 146 & 3,88 & 100 & 2,17 & 0,43 & 2,71 & 8,77 \\
\hline Eugenia aurata O. Berg & Myrtaceae & 131 & 3,48 & 100 & 2,17 & 0,38 & 2,44 & 8,10 \\
\hline Erythroxylum tortuosum Mart. & Erythroxylaceae & 137 & 3,64 & 100 & 2,17 & 0,17 & 1,05 & 6,87 \\
\hline Dalbergia miscolobium Benth. & Fabaceae & 112 & 2,97 & 70 & 1,52 & 0,28 & 1,76 & 6,26 \\
\hline Tachigali aurea Tul. & Fabaceae & 81 & 2,15 & 100 & 2,17 & 0,29 & 1,83 & 6,16 \\
\hline Syagrus flexuosa (Mart.) Becc.** & Arecaceae & 99 & 2,63 & 80 & 1,74 & 0,28 & 1,75 & 6,13 \\
\hline Pterodon pubescens (Benth.) Benth. & Fabaceae & 36 & 0,96 & 90 & 1,96 & 0,49 & 3,10 & 6,01 \\
\hline Hymenaea stigonocarpa Mart. ex Hayne & Fabaceae & 68 & 1,81 & 100 & 2,17 & 0,31 & 1,97 & 5,96 \\
\hline Davilla elliptica A. St.-Hil. & Dilleniaceae & 80 & 2,12 & 100 & 2,17 & 0,21 & 1,35 & 5,65 \\
\hline Qualea multiflora Mart. & Vochysiaceae & 57 & 1,51 & 90 & 1,96 & 0,32 & 2,01 & 5,49 \\
\hline Lafoensia pacari A. St.-Hil. & Lythraceae & 76 & 2,02 & 100 & 2,17 & 0,20 & 1,28 & 5,48 \\
\hline Leptolobium dasycarpum Vogel & Fabaceae & 87 & 2,31 & 80 & 1,74 & 0,18 & 1,16 & 5,22 \\
\hline Magonia pubescens A. St.-Hil. & Sapindaceae & 41 & 1,09 & 70 & 1,52 & 0,36 & 2,30 & 4,91 \\
\hline Myrcia lanuginosa $\mathrm{O}$. Berg & Myrtaceae & 77 & 2,04 & 90 & 1,96 & 0,13 & 0,85 & 4,86 \\
\hline Aspidosperma macrocarpon Mart. & Apocynaceae & 32 & 0,85 & 90 & 1,96 & 0,27 & 1,71 & 4,52 \\
\hline Mezilaurus crassiramea (Meisn.) Taub. ex Mez & Lauraceae & 26 & 0,69 & 80 & 1,74 & 0,27 & 1,71 & 4,15 \\
\hline Arrabidaea cinnamomea (A. DC.) Sandwith * & Bignoniaceae & 41 & 1,09 & 100 & 2,17 & 0,08 & 0,51 & 3,78 \\
\hline Byrsonima coccolobifolia Kunth & Malpighiaceae & 26 & 0,69 & 100 & 2,17 & 0,09 & 0,58 & 3,45 \\
\hline Ouratea spectabilis (Mart. ex Engl.) Engl. & Ochnaceae & 19 & 0,50 & 80 & 1,74 & 0,18 & 1,11 & 3,36 \\
\hline Aspidosperma tomentosum Mart. & Apocynaceae & 31 & 0,82 & 70 & 1,52 & 0,16 & 0,99 & 3,34 \\
\hline Qualea grandiflora Mart. & Vochysiaceae & 19 & 0,50 & 80 & 1,74 & 0,16 & 1,00 & 3,25 \\
\hline Guapira graciliflora (Schmidt) Lundell & Nyctaginaceae & 25 & 0,66 & 90 & 1,96 & 0,09 & 0,57 & 3,20 \\
\hline Plathymenia reticulata Benth. & Fabaceae & 26 & 0,69 & 70 & 1,52 & 0,15 & 0,95 & 3,17 \\
\hline Tocoyena formosa (Cham. \& Schltdl.) K. Schum. & Rubiaceae & 34 & 0,90 & 80 & 1,74 & 0,07 & 0,44 & 3,09 \\
\hline Bowdichia virgilioides Kunth & Fabaceae & 17 & 0,45 & 60 & 1,30 & 0,19 & 1,23 & 2,99 \\
\hline Cordiera sessilis (Vell.) Kuntze & Rubiaceae & 19 & 0,50 & 80 & 1,74 & 0,09 & 0,61 & 2,85 \\
\hline Mouriri elliptica Mart. & Melastomataceae & 16 & 0,42 & 70 & 1,52 & 0,12 & 0,77 & 2,72 \\
\hline Eriotheca gracilipes (K. Schum.) A. Robyns & Malvaceae & 24 & 0,64 & 70 & 1,52 & 0,06 & 0,39 & 2,55 \\
\hline Vellozia sp. ** & Velloziaceae & 40 & 1,06 & 40 & 0,87 & 0,09 & 0,58 & 2,52 \\
\hline Kielmeyera coriacea Mart. \& Zucc. & Clusiaceae & 20 & 0,53 & 70 & 1,52 & 0,05 & 0,34 & 2,39 \\
\hline Eugenia glazioviana Kiaersk. & Myrtaceae & 11 & 0,29 & 60 & 1,30 & 0,11 & 0,67 & 2,27 \\
\hline Erythroxylum engleri O.E. Schulz & Erythroxylaceae & 21 & 0,56 & 70 & 1,52 & 0,03 & 0,20 & 2,27 \\
\hline Byrsonima pachyphylla A. Juss. & Malpighiaceae & 17 & 0,45 & 50 & 1,09 & 0,09 & 0,57 & 2,11 \\
\hline Tabebuia aurea (S. Manso) Benth. \& H. f. ex S. Moore & Bignoniaceae & 13 & 0,35 & 40 & 0,87 & 0,14 & 0,86 & 2,07 \\
\hline
\end{tabular}


Tabela 1. Continuação...

\begin{tabular}{|c|c|c|c|c|c|c|c|c|}
\hline Espécie & Família & DA & DR & FA & FR & DoA & DoR & IVI \\
\hline Eugenia gemmiflora $\mathrm{O}$. Berg & Myrtaceae & 11 & 0,29 & 60 & 1,30 & 0,07 & 0,43 & 2,02 \\
\hline Andira cuiabensis Benth. & Fabaceae & 7 & 0,19 & 40 & 0,87 & 0,14 & 0,89 & 1,94 \\
\hline Agonandra brasiliensis Miers ex Benth. \& Hook. f. & Opiliaceae & 18 & 0,48 & 50 & 1,09 & 0,05 & 0,28 & 1,84 \\
\hline Connarus suberosus Planch. & Connaraceae & 16 & 0,42 & 50 & 1,09 & 0,03 & 0,18 & 1,69 \\
\hline Mimosa laticifera Rizzini \& A. Mattos & Fabaceae & 21 & 0,56 & 40 & 0,87 & 0,04 & 0,25 & 1,67 \\
\hline Rourea induta Planch. & Connaraceae & 18 & 0,48 & 50 & 1,09 & 0,02 & 0,12 & 1,68 \\
\hline Salvertia convallariodora A. St.-Hil. & Vochysiaceae & 9 & 0,24 & 30 & 0,65 & 0,11 & 0,72 & 1,60 \\
\hline Curatella americana $\mathrm{L}$. & Dilleniaceae & 6 & 0,16 & 40 & 0,87 & 0,09 & 0,55 & 1,57 \\
\hline Casearia sylvestris $\mathrm{Sw}$. & Salicaceae & 9 & 0,24 & 60 & 1,30 & 0,01 & 0,05 & 1,59 \\
\hline Pouteria ramiflora (Mart.) Radlk. & Sapotaceae & 6 & 0,16 & 40 & 0,87 & 0,07 & 0,47 & 1,49 \\
\hline Himatanthus obovatus (Müll. Arg.) Woodson & Apocynaceae & 12 & 0,32 & 40 & 0,87 & 0,01 & 0,09 & 1,28 \\
\hline Syagrus comosa (Mart.) Mart.** & Arecaceae & 7 & 0,19 & 40 & 0,87 & 0,04 & 0,24 & 1,29 \\
\hline Myrcia tomentosa (Aubl.) DC. & Myrtaceae & 5 & 0,13 & 40 & 0,87 & 0,03 & 0,19 & 1,19 \\
\hline Buchenavia tomentosa Eichler & Combretaceae & 3 & 0,08 & 30 & 0,65 & 0,07 & 0,44 & 1,17 \\
\hline Banisteriopsis pubipetala (A. Juss.) Cuatrec.* & Malpighiaceae & 6 & 0,16 & 40 & 0,87 & 0,01 & 0,10 & 1,12 \\
\hline Annona coriacea Mart. & Annonaceae & 6 & 0,16 & 40 & 0,87 & 0,02 & 0,12 & 1,15 \\
\hline Antonia ovata Pohl & Loganiaceae & 11 & 0,29 & 30 & 0,65 & 0,03 & 0,18 & 1,12 \\
\hline Ouratea hexasperma (A. St.-Hil.) Baill. & Ochnaceae & 5 & 0,13 & 40 & 0,87 & 0,02 & 0,10 & 1,10 \\
\hline Jacaranda brasiliana (Lam.) Pers. & Bignoniaceae & 6 & 0,16 & 20 & 0,43 & 0,08 & 0,50 & 1,09 \\
\hline Pouteria gardneri (Mart. \& Miq.) Baehni & Sapotaceae & 6 & 0,16 & 20 & 0,43 & 0,08 & 0,48 & 1,07 \\
\hline Luetzelburgia praecox (Harms ex Kuntze) Harms & Fabaceae & 5 & 0,13 & 30 & 0,65 & 0,05 & 0,30 & 1,08 \\
\hline Astronium fraxinifolium Schott ex Spreng. & Anacardiaceae & 10 & 0,27 & 30 & 0,65 & 0,02 & 0,15 & 1,06 \\
\hline Dimorphandra mollis Benth. & Fabaceae & 5 & 0,13 & 30 & 0,65 & 0,01 & 0,07 & 0,85 \\
\hline Erythroxylum testaceum Peyr. & Erythroxylaceae & 5 & 0,13 & 30 & 0,65 & 0,01 & 0,04 & 0,82 \\
\hline Emmotum nitens (Benth.) Miers & Icacinaceae & 2 & 0,05 & 20 & 0,43 & 0,05 & 0,30 & 0,79 \\
\hline Protium heptaphyllum (Aubl.) Marchand & Burseraceae & 2 & 0,05 & 20 & 0,43 & 0,02 & 0,10 & 0,58 \\
\hline Copaifera langsdorffii Desf. & Fabaceae & 1 & 0,03 & 10 & 0,22 & 0,05 & 0,31 & 0,55 \\
\hline Cordiera elliptica Kuntze & Rubiaceae & 3 & 0,08 & 20 & 0,43 & 0,01 & 0,03 & 0,54 \\
\hline Simarouba versicolor A. St.-Hil. & Simaroubaceae & 3 & 0,08 & 20 & 0,43 & 0,01 & 0,02 & 0,53 \\
\hline Peritassa campestris (Cambess.) A.C. Sm. & Celastraceae & 2 & 0,05 & 20 & 0,43 & 0,01 & 0,03 & 0,51 \\
\hline Eugenia multiflora Lam. & Myrtaceae & 2 & 0,05 & 20 & 0,43 & 0,01 & 0,01 & 0,49 \\
\hline Neea theifera Oerst. & Nyctaginaceae & 7 & 0,19 & 10 & 0,22 & 0,01 & 0,06 & 0,46 \\
\hline Heisteria ovata Benth. & Olacaceae & 3 & 0,08 & 10 & 0,22 & 0,01 & 0,09 & 0,39 \\
\hline Strychnos pseudoquina A. St.-Hil. & Loganiaceae & 1 & 0,03 & 10 & 0,22 & 0,02 & 0,11 & 0,35 \\
\hline Cybistax antisyphilitica (Mart.) Mart. & Bignoniaceae & 2 & 0,05 & 10 & 0,22 & 0,01 & 0,03 & 0,29 \\
\hline Ferdinandusa elliptica Pohl & Rubiaceae & 1 & 0,03 & 10 & 0,22 & 0,01 & 0,06 & 0,30 \\
\hline Hancornia speciosa B.A. Gomes & Apocynaceae & 1 & 0,03 & 10 & 0,22 & 0,01 & 0,03 & 0,27 \\
\hline Brosimum gaudichaudii Trécul & Moraceae & 1 & 0,03 & 10 & 0,22 & 0,01 & 0,01 & 0,25 \\
\hline Diospyros hispida A. DC. & Ebenaceae & 2 & 0,05 & 10 & 0,22 & 0,01 & 0,01 & 0,28 \\
\hline Plenckia populnea Reissek & Celastraceae & 1 & 0,03 & 10 & 0,22 & 0,01 & 0,02 & 0,26 \\
\hline Matayba guianensis Aubl. & Sapindaceae & 1 & 0,03 & 10 & 0,22 & 0,01 & 0,01 & 0,24 \\
\hline Eugenia punicifolia (Kunth) DC. & Myrtaceae & 1 & 0,03 & 10 & 0,22 & 0,01 & 0,01 & 0,25 \\
\hline Aspidosperma subincanum Mart. & Apocynaceae & 1 & 0,03 & 10 & 0,22 & 0,01 & 0,01 & 0,24 \\
\hline Machaerium acutifolium Vogel & Fabaceae & 1 & 0,03 & 10 & 0,22 & 0,01 & 0,01 & 0,25 \\
\hline Total & & 3.766 & 100 & 4.660 & 100 & 15,72 & 100 & 300 \\
\hline
\end{tabular}


Tabela 2. Comparações florísticas e estruturais entre a comunidade lenhosa com DAS $\geq 3 \mathrm{~cm}$ (primeira linha) e DAS $\geq 3 \mathrm{~cm}$ (segunda linha) do cerrado rupestre do Parque Municipal do Bacaba, na transição entre os biomas Cerrado e Floresta Amazônica, Mato Grosso, e outros estudos com comunidades lenhosas de cerrado rupestre e típico do bioma Cerrado (DAS $\geq 5 \mathrm{~cm}$ ). N = número de espécies; $\mathrm{H}^{\prime}=$ índice de diversidade de espécies de Shannon-Wiener (H'); J = equabilidade de Pielou, $\mathrm{D}=$ densidade (ind.ha $\left.{ }^{-1}\right) ; \mathrm{AB}=$ área basal $\left(\mathrm{m}^{2} \cdot \mathrm{ha}^{-1}\right)$. Dist. = Distância $(\mathrm{km})$ em linha reta do CRPB até as demais áreas analisadas; Alt.= altitude.

Table 2. Floristic and structural comparisons among the woody community with DSH $\geq 3 \mathrm{~cm}$ (first line) and DSH $\geq 5 \mathrm{~cm}$ (second line) of the "cerrado rupestre" at Bacaba's Park, in the Cerrado-Amazon forest transition zone of eastern Mato Grosso, and other studies with typical cerrado and "cerrado rupestre" communities within the Cerrado biome. $\mathrm{N}=$ Species number; $\mathrm{H}^{\prime}=$ Shannon-Wiener index of species diversity $\left(\mathrm{H}^{\prime}\right)$; $\mathrm{J}=$ Pielou equability index, $\mathrm{D}=$ density (ind.ha $\left.{ }^{-1}\right) ; \mathrm{AB}=$ basal area $\left(\mathrm{m}^{2} \cdot \mathrm{ha}^{-1}\right)$. Dist. $=$ Distance $(\mathrm{km})$ measured in a straight line from CRPB to other compared areas; Alt. $=$ altitude.

\begin{tabular}{|c|c|c|c|c|c|c|c|c|}
\hline Autores & Estado & $\mathbf{N}$ & $\mathbf{H}^{\prime}$ & $\mathbf{J}$ & D & $\mathbf{A B}$ & Dist. & Alt. \\
\hline \multicolumn{9}{|l|}{ Cerrado Rupestre/Neossolo Litólico } \\
\hline Presente estudo & MT & 85 & 3,45 & 0,77 & 3.766 & 15,72 & - & 400 \\
\hline & & 74 & 3,59 & 0,83 & 1.572 & 13,27 & & \\
\hline Miranda et al. (2007) & GO & 54 & 3,13 & 0,79 & 1.137 & 7,08 & 276 & 800 \\
\hline Lima et al. (2010) & GO & 66 & 3,33 & 0,79 & 1.357 & 12,39 & 520 & 1.000 \\
\hline Pinto et al. (2009) & GO & 65 & 3,45 & 0,83 & 674 & 5,67 & 433 & 1.200 \\
\hline Moura et al. (2007) & GO & 56 & 3,33 & 0,82 & 461 & 3,43 & 417 & 1.385 \\
\hline Amaral et al. (2006) & DF & 51 & 3,08 & - & 607 & 3,58 & 520 & 1.050 \\
\hline \multicolumn{9}{|l|}{ Cerrado Típico/Latossolo } \\
\hline Marimon Jr. \& Haridasan (2005) & MT & 77 & 3,78 & 0,87 & 1.890 & 14,94 & 2 & 250 \\
\hline Nogueira et al. (2001) & MT & 88 & 3,78 & 0,84 & 1.250 & 9,36 & 130 & 400 \\
\hline Felfili et al. (2002) & MT & 73 & 3,69 & 0,84 & 947 & 7,09 & 74 & 500 \\
\hline Balduino et al. (2005) & MG & 73 & 3,57 & 0,83 & 1.813 & 16,42 & 1.010 & 750 \\
\hline Assunção \& Felfili (2004) & DF & 54 & 3,41 & - & 818 & 8,97 & 520 & 1.050 \\
\hline
\end{tabular}

relação à distribuição diamétrica dos indivíduos vivos na comunidade, foi observada uma tendência de diminuição nas frequências das classes menores para as maiores, em um padrão de J-reverso. A maioria dos indivíduos $(59,1 \%)$ apresentou troncos com DAS entre 3 e $5 \mathrm{~cm}$. Nessa classe diamétrica foram amostradas 78 espécies (91,7\% do total) (Figura 2b).

A análise de classificação (UPGMA) revelou a existência de dois grandes grupos. O primeiro formado por áreas de cerrado rupestre e típico em locais com altitudes inferiores a $800 \mathrm{~m}$ e o segundo por cerrados rupestres e típicos localizados no planalto central brasileiro com altitudes superiores a $1.000 \mathrm{~m}$. Notou-se ainda que o primeiro grupo foi dividido em: áreas do estado de Mato Grosso de baixa altitude (inferiores a $500 \mathrm{~m}$ ) e próximos a transição com a Floresta Amazônica; áreas do estado de Goiás com altitudes intermediárias (entre 600 e 800 m) e uma área no estado de Minas Gerais com altitude intermediária $(750 \mathrm{~m})$ e nas proximidades com a Mata Atlântica (Figura 3).

Comparando-se os dois critérios mínimos de inclusão adotados no presente estudo (DAS $\geq 3 \mathrm{~cm}$ e DAS $\geq 5 \mathrm{~cm}$ ), pode-se notar que o primeiro aumentou o número de espécies registradas e os valores de densidade e de área basal total da comunidade estudada (Tabela 2).

\section{Discussão}

A riqueza registrada e estimada de espécies, o índice de diversidade de espécies e a equabilidade encontrados no cerrado rupestre estudado foram elevados e similares àqueles registrados em outros estudos com cerrado típico no leste mato-grossense (Marimon et al. 1998, Felfili et al. 2002, Marimon Jr. \& Haridasan 2005). A suficiência do esforço amostral empregado $\left(10.000 \mathrm{~m}^{2}\right)$ no presente estudo, revelada pelo método de rarefação, confirma os resultados dos estudos realizados por Assunção \& Felfili (2004) e Andrade et al. (2002), em cerrado sentido restrito, que também indicaram que amostragens em áreas superiores a $7.000 \mathrm{~m}^{2}$ já foram suficientes para registrar cerca de $90 \%$ das espécies.
Apesar das limitações impostas pelo substrato rochoso ao estabelecimento das plantas, o cerrado rupestre estudado apresentou fortes semelhanças em termos de riqueza, diversidade, bem como as mais altas similaridades florísticas, com três outras áreas de cerrado sobre Latossolos do leste mato-grossense, sugerindo que a proximidade geográfica pode ter forte influência sobre a riqueza e a composição de espécies na região. Este aspecto foi confirmado pela análise de classificação que agrupou as quatro áreas de cerrado do leste mato-grossense, independente do tipo fisionômico e do substrato. O agrupamento de áreas com altitudes semelhantes também sugere a influência da altitude sobre a composição da flora lenhosa de formações savânicas de Cerrado, corroborando a afirmação de Castro et al. (1999). Os padrões florísticos aqui encontrados enfatizam a importância dos ambientes rupestres na manutenção da riqueza e composição florística da vegetação savânica do leste mato-grossense, uma vez que áreas rupestres são mantidas em função de serem inadequadas para a mecanização e implantação de atividades agropecuárias convencionais.

A diversidade florística registrada neste estudo foi superior à observada em outros estudos realizados em comunidades lenhosas de cerrado rupestre no planalto central (Amaral et al. 2006, Moura et al. 2007, Pinto et al. 2009, Lima et al. 2010). A notável riqueza e diversidade de espécies e as mais elevadas semelhanças florísticas entre as fitofisionomias savânicas do leste mato-grossense, podem estar relacionadas com a localização pré-Amazônica da área estudada. Segundo Felfili et al. (2002), a influência florística da Amazônica contribui com estes elevados índices de diversidade e riqueza de espécies na região de transição entre os biomas Cerrado e Floresta Amazônica.

Espécies habitat-especialistas de cerrados rupestres, como Wunderlichia cruelsiana Taub. (Munhoz \& Proença 1998, Miranda et al. 2007, Amaral et al. 2006), Wunderlichia mirabilis Riedel ex Baker (Pinto et al. 2009), Tibouchina papyrus (Pohl) Toledo, Swartzia adamantium (Cambess.) Bedell ex Giraldo-Cañas e Clusia burchelli Engl. (Moura et al. 2007, Pinto et al. 2009) não 


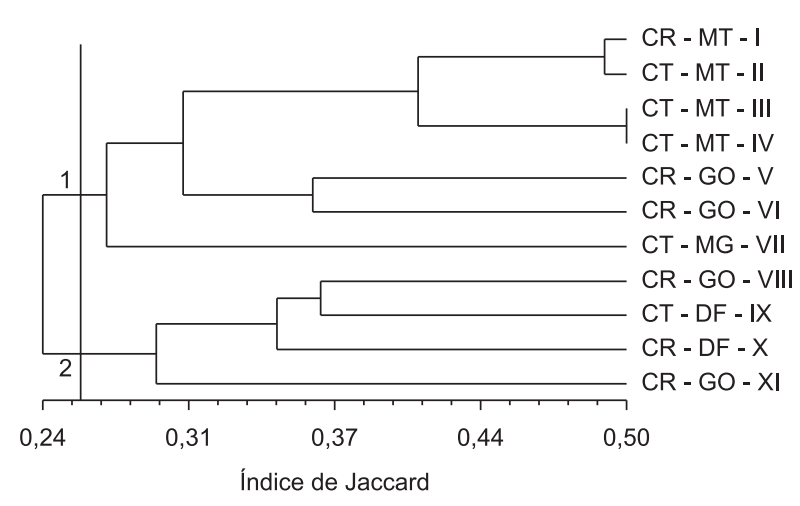

Figura 3. Dendrograma de similaridade florística entre comunidades lenhosas (DAS $\geq 5 \mathrm{~cm}$ ) obtido pelo método de ligação UPGMA (Coeficiente de Valor Cofenetico = 0,77). $\mathrm{I}=$ presente estudo, II = Marimon Jr. \& Haridasan (2005), III = Nogueira et al. (2001), IV = Felfili et al. (2002), V = Miranda et al. (2007), VI = Lima et al. (2010), VII = Balduino et al. (2005), VIII = Pinto et al. (2009), IX = Assunção \& Felfili (2004), X = Amaral et al. (2006), XI = Moura et al. (2007).

Figure 3. Dendrogram of floristic similarity between woody communities (DSH $\geq 5 \mathrm{~cm}$ ) using the UPGMA (Cofenetic Coefficient $=0,77$ ) linkage method. I = this study, II = Marimon Jr. \& Haridasan (2005), III = Nogueira et al. (2001), IV = Felfili et al. (2002), V = Miranda et al. (2007), VI = Lima et al. (2010), VII = Balduino et al. (2005), VIII = Pinto et al. (2009), IX = Assunção \& Felfili (2004), X = Amaral et al. (2006), XI = Moura et al. (2007).

foram registradas no cerrado rupestre estudado. Segundo Rabinowitz (1981), espécies habitat-especialistas apresentam elevada importância estrutural e por isso podem ser consideradas como espécies indicadoras de determinados ambientes. Munhoz \& Proença (1998) acreditam que o endemismo e a especificidade observada em espécies de ambientes rupestres do Cerrado podem também estar relacionados a altitudes mais elevadas. Entre os cerrados rupestres amostrados até o momento, apenas o do presente estudo possui altitudes inferiores a 400 m, enquanto os demais (Amaral et al. 2006, Moura et al. 2007, Pinto et al. 2009, Lima et al. 2010) se localizam acima de 700 m, corroborando em parte com a afirmação de Munhoz \& Proença (1998). Assim, sugerimos que os endemismos nos cerrados rupestres podem ser determinados tanto pela altitude quanto pela adaptação ou especialização de algumas espécies aos substratos rochosos.

A ampla variação nos valores de densidade e área basal registrada nas áreas de cerrado rupestre e típico aqui comparadas sugere que as propriedades estruturais das comunidades savânicas de cerrado parecem depender de diferentes fatores físicos locais, conforme sugerido por Ribeiro \& Walter (2008). No entanto, não foram observadas tendências para cerrados sobre Latossolos apresentarem densidades e áreas basais maiores das observadas em cerrados rupestres. Assim, esses dois parâmetros estruturais da vegetação parecem não depender apenas da suposta limitação do substrato rochoso ao estabelecimento das plantas lenhosas em ambientes rupestres de cerrado.

Os valores de densidade e área basal, bem como a riqueza florística do cerrado rupestre estudado foram fortemente influenciados pelo critério mínimo de inclusão adotado. Esse fato sugere que estudos que adotam $5 \mathrm{~cm}$, ao invés de $3 \mathrm{~cm}$ de diâmetro, como critério mínimo de inclusão, subestimam a biomassa e diversidade alfa de comunidades savânicas, pois excluem indivíduos de espécies arbustivas e subarbustivas. Estudos florísticos e fitossociológicos em fitofisionomias savânicas do bioma Cerrado geralmente excluem monocotiledôneas (Lima et al. 2003) e lianas lenhosas (Fidelis \& Godoy 2003), grupos esses que apresentaram elevada importância florística e estrutural na comunidade aqui estudada. Dessa forma, recomenda-se que estudos futuros incluam lianas e monocotiledôneas e a adotem limite mínimo de inclusão de $3 \mathrm{~cm}$ para garantir uma melhor caracterização das comunidades.

$\mathrm{O}$ percentual de indivíduos mortos em pé registrado no presente estudo $(3 \%)$ foi semelhante àqueles registrados em cerrado sentido restrito por Felfili et al. (2002) (5\%) e em cerrado rupestre por Amaral et al. (2006) (3,8\%). Segundo Felfili \& Fagg (2007), valores em torno de $5 \%$ de indivíduos mortos em cerrado sentido restrito indicam baixo distúrbio na comunidade. Sendo assim, o número reduzido de indivíduos mortos aqui amostrados sugere que a área encontra-se bem protegida de interferências antrópicas, especialmente do fogo.

A importância estrutural de Erythroxylum suberosum, registrada no presente estudo, também têm sido destacada em outros levantamentos de cerrado sentido restrito (Marimon et al. 1998, Felfili et al. 2002, Balduino et al. 2005), cerrado rupestre (Amaral et al. 2006, Felfili \& Fagg 2007, Abad et al. 2010) e campos de murundus (Marimon et al. 2008). A espécie Qualea parviflora configura entre as 10 espécies de maior VI em estudos realizados em cerrado sentido restrito (Marimon et al. 1998, Nogueira et al. 2001, Felfili et al. 2002, Silva et al. 2002, Balduino et al. 2005, Marimon Jr. \& Haridasan 2005, Felfili \& Fagg 2007), cerrado rupestre (Pinto et al. 2009, Lima et al. 2010) e campo de murundu (Marimon et al. 2008). Essas duas espécies são citadas por Ratter et al. (2003) como apresentando ampla distribuição no Cerrado, fato que poderia ser explicado pela adaptação dessas espécies a diferentes ambientes e substratos.

A proporção de espécies raras no presente estudo $(11,8 \%)$ está dentro dos limites registrados em áreas de cerrado típico, que variam entre 9,1 e 19,7\% (Marimon et al. 1998, Nogueira et al. 2001, Felfili et al. 2002, Assunção \& Felfili 2004, Marimon Jr. \& Haridasan 2005). Apesar disso, outros levantamentos realizados em cerrados rupestres do bioma registraram maior número de espécies raras, com proporções variando de 20 a 39\% (Amaral et al. 2006, Moura et al. 2007, Pinto et al. 2009, Abad et al. 2010). Segundo Pinto et al. (2009), a raridade em ambientes rupestres é elevada em função das baixas densidades apresentadas por espécies restritas a essa fitofisionomia. Entretanto, esse não foi o caso da comunidade estudada, que não apresentou espécies com especificidade de hábitats rupestres.

A distribuição das classes de alturas do cerrado rupestre estudado apresentou padrão unimodal tendendo para distribuição normal, de modo similar ao que já foi registrado em outras áreas de cerrado rupestre (Amaral et al. 2006, Pinto et al. 2009, Lima et al. 2010) e típico (Assunção \& Felfili 2004, Marimon Jr. \& Haridasan 2005) do Brasil Central. Nessas comunidades, as estrutura vertical é representada predominantemente por espécies arbustivas, ou seja, com alturas entre 1 e $3 \mathrm{~m}$, caracterizando assim uma vegetação tipicamente savânica.

A distribuição diamétrica na comunidade estudada seguiu o modelo de J-reverso, comumente registrado em comunidades savânicas bem preservadas do bioma Cerrado (Felfili \& Silva-Júnior 1988, Assunção \& Felfili 2004), incluindo cerrados rupestres (Amaral et al. 2006, Moura et al. 2007, Pinto et al. 2009). Segundo Felfili \& Silva-Júnior (1988), esse padrão sugere um recrutamento contínuo e um equilíbrio entre mortalidade e recrutamento na comunidade, garantindo a manutenção da atual estrutura diamétrica na ausência de distúrbios. Provavelmente a localização da área estudada em uma unidade de conservação sujeita a poucos distúrbios pode estar contribuindo com a distribuição diamétrica apresentada pelos indivíduos amostrados.

Esse e outros poucos estudos desenvolvidos com a flora lenhosa em cerrado rupestre aqui considerados evidenciam elevada riqueza e diversidade de espécies. Além disso, os parâmetros florísticos e estruturais chamam atenção para a importância dos ambientes 
rupestres na manutenção da biodiversidade do Cerrado e para a condução de estudos que adotem em sua amostragem diâmetros mínimos de $3 \mathrm{~cm}$, sob o risco de se retratar apenas parcialmente a real estrutura da flora lenhosa dessa peculiar e rara fitofisionomia do bioma Cerrado, uma vez que cerca de $60 \%$ dos indivíduos apresentaram DAS inferiores a $5 \mathrm{~cm}$. Finalmente, a posição geográfica da área de estudo, na transição entre os biomas Cerrado e Floresta Amazônica, bem como o bom estado atual de conservação da área, localizada em uma unidade de conservação, podem explicar a elevada biodiversidade e área basal e a estabilidade estrutural da comunidade.

\section{Agradecimentos}

Aos biólogos MSc. Daniel Franczak, Leonardo Maracahipes, MSc. Tassiana Santos, Ludimila Pinheiro, Edson Pinto, Kátia Martins, Gaildes Constantino e o agrônomo Divino Pereira pelo auxílio no trabalho de campo. À Universidade do Estado de Mato Grosso UNEMAT, Campus de Nova Xavantina, pela concessão de bolsa de estudos e pelo material disponibilizado. Ao Programa PELD/CAPES (Proc. $n^{\circ}$ 558069/2009-6) pelo apoio financeiro. À FAPEMAT pela concessão de bolsa de estudos. Aos revisores anônimos, pelas valiosas críticas e sugestões.

\section{Referências Bibliográficas}

ABAD, J.C.S. \& MARIMON, B.S. 2008. Caracterização e diagnóstico ambiental do Parque Municipal do Bacaba (Nova Xavantina, MT). In Gestão e educação ambiental: água, biodiversidade e cultura (J.E. Santos \& C. Galbiati, orgs.). Rima, São Carlos, v.1, p.23-56.

ABAD, J.C.S., MARIMON, B.S., JANCOSKI, H.S., SILVA, L.S., OLIVEIRA, E.A. \& JORDÃO, B.O. 2010. Composição florística e estrutural das comunidades lenhosas de cerrado rupestre e de florestas de galeria do parque Municipal do Bacaba, Nova Xavantina, MT. In Gestão e Educação Ambiental - Água, Biodiversidade e Cultura (J.E. Santos, C. Galbiati \& L.E. Moschini, org.). Rima, São Carlos, v.2, p.82-102.

ALVES, R.J.V. \& KOLBEK, J. 2010. Can campo rupestre vegetation be floristically delimited based on vascular plant genera? Plant. Ecol. 207:67-79.

AMArAl, A.G., PEREIRA, F.F.O. \& MUNHOZ, C.B.R. 2006. Fitossociologia de uma área de cerrado rupestre na Fazenda Sucupira, Brasília-DF. Cerne 12(4):350-359.

ANDRADE, L.A.Z, FELFILI, J.M. \& VIOLATTI, L. 2002. Fitossociologia de uma área de cerrado denso na RECOR-IBGE, Brasília-DF. Acta Bot. Bras. 16(2):225-240.

ANGIOSPERM PHYLOGENY GROUP - APG. 2009. An update of the Angiosperm Phylogeny Group classification for the orders and families of flowering plants: APG III. Bot. J. Linn. Soc. 161(2):105-121.

ASSUNÇÃO, S.L. \& FELFILI, J.M. 2004. Fitossociologia de um fragmento de cerrado sensu stricto na APA do Paranoá, DF, Brasil. Acta Bot. Bras. 18(4):903-909.

BALDUINO, A.P.C., SOUZA, A.L., MEIRA-NETO, J.A.A., SILVA, A.F. \& SILVA-Jr., M.C. 2005. Fitossociologia e análise comparativa da composição florística do cerrado da flora de Paraopeba-MG. Rev. Árvore 29(1):25-34.

BRIDGEWATER, S., RATTER, J.A. \& RIBEIRO, J.F. 2004. Biogeographic patterns, b-diversity and dominance in the cerrado biome of Brazil. Biodiv. Conserv. 13:2295-2318.

BROWER, J.E. \& ZAR, J.H. 1977. Field and laboratory methods for general ecology. W. C. Brown Co. Pub., Iowa.

CAMARGO, A.P. 1963. Clima do Cerrado. In Simpósio sobre o Cerrado (M.G. Ferri, coord.). EDUSP, São Paulo, p.75-95.

CASTRO, A.A.J.F., MARTINS, F.R., TAMASHIRO, J.Y. \& SHEPHERD, G.J. 1999. How rich is the flora of brazilian cerrados? Ann. Mo. Bot. Gard. 86(1):192-224.

CIENTEC. 2006. Mata Nativa 2: manual do usuário. Cientec,Viçosa.
COLWELL, R.K. 2008. EstimateS: Statistical estimation of species richness and shared species from samples. Version 8.0. http://www.purl.oclc.org/ estimates (último acesso em 15/11/2008).

EITEN, G. 1979. Formas fisionômicas do Cerrado. Rev. Bras. Bot. 2:139-148.

FELFILI, J.M. \& FAGG, C.W. 2007. Floristic composition, diversity and structure of the "cerrado" sensu stricto on rocky soils in northern Goiás and southern Tocantins, Brazil. Rev. Bras. Bot. 30(3):375-385.

FELFILI, M.C. \& FELFILI, J.M. 2001. Diversidade alfa e beta no cerrado sensu stricto da Chapada Pratinha, Brasil. Acta Bot. Bras. 15(2):243-254.

FELFILI, J.M. \& SILVA-Jr., M.C. 1988. Distribuição dos diâmetros numa faixa de Cerrado na Fazenda Água Limpa (FAL) em Brasília-DF. Acta Bot. Bras. 2(1-2):85-104.

FELFILI, J.M., CARVALHO, F.A. \& HAIDAR, R.F. 2005. Manual para o monitoramento de parcelas permanentes nos biomas Cerrado e Pantanal. EdUnB, Brasília, 55p.

FELFILI, J.M., NOGUEIRA, P.E., SILVA-Jr., M.C., MARIMON, B.S. \& DELITTI, W.B.C. 2002. Composição florística e fitossociológica do cerrado sentido restrito no município de Água Boa-MT. Acta Bot. Bras. 16(1):103-112.

FIDELIS, A.T. \& GODOY, S.A.P. 2003. Estrutura de um cerrado stricto sensu na Gleba Cerrado Pé-de-Gigante, Santa Rita do Passa Quatro, SP. Acta Bot. Bras. 17(4):531-539.

GOTELLI, N.J. \& COLWELL, R.K. 2001. Quantifying biodiversity: procedures and pitfalls in the measurement and comparison of species richness. Ecol. Lett. 4:379-391.

KENT, M. \& COKER, P. 1992. Vegetation description and analysis; a practical approach. Belhaven Press, London.

KLINK, C.A. \& MACHADO, R.B. 2005. Conservation of the Brazilian Cerrado. Conserv. Biol. 19(3):707-713.

LIMA, E.S., FELFILI, J.M., MARIMON, B.S. \& SCARIOT, A. 2003. Diversidade, estrutura e distribuição espacial de palmeiras em um cerrado sensu stricto no Brasil Central-DF. Rev. Bras. Bot. 26(3):361-370.

LIMA, T.A., PINTO, J.R.R., LENZA, E. \& PINTO, A.S. 2010. Florística e estrutura da vegetação arbustivo-arbórea em uma área de cerrado rupestre no Parque Estadual da Serra de Caldas Novas, Goiás. Biota Neotrop. 10(2): http://www.biotaneotropica.org.br/v10n2/en/ abstract?article+bn04010022010.

LUDWING, J.A. \& REYNOLDS, J.E. 1988. Statistical ecology - a primer on methods and computing. J. Wiley \& Sons, New York.

MACHADO, R.B., RAMOS NETO, M.B., PEREIRA, P.G.P., CALDAS, E.F., GONÇALVES, D.A., SANTOS, N.S., TABOR, K. \& STEININGER, M. 2004. Estimativas de perda da área do Cerrado brasileiro. Conservação Internacional - Brasil, Brasília.

MAGURRAN, A.E. 1988. Ecological diversity and its measurement. Croom Helm., London.

MARIMON, B.S., LIMA, E.S., DUARTE, T.G., CHIEREGATTO, L.C. \& RATTER, J.A. 2006. Observations on the vegetation of northeastern Mato Grosso, Brazil. IV. An analysis of the Cerrado-Amazonian Forest ecotone. Edinb. J. Bot. 63(23):323-341.

MARIMON, B.S., IVANAUSKAS, N.M., FERNANDES-BULHÃO, C. \& LIMA, E.S. 2008. Caracterização florística e fitossociologica da planície de inundação do Rio das Mortes, MT. In Fauna e flora da planície de inundação do Rio das Mortes - MT (H.S.R. Cabette, Org.). UNEMAT, Cáceres, p.15-30.

MARIMON, B.S., VARELLA, R.F. \& MARIMON Jr., B.H. 1998. Fitossociologia de uma área de cerrado de encosta em Nova Xavantina, Mato Grosso. Bol. Herb. Ezechias Paulo Heringer 3:82-101.

MARIMON Jr., B.H. \& HARIDASAN, M. 2005. Comparação da vegetação arbórea e características edáficas de um cerradão e um cerrado sensu stricto em áreas adjacentes sobre solo distrófico no leste de Mato Grosso, Brasil. Acta Bot. Bras. 19(4):913-926.

MARTINELLI, G. 2007. Mountain biodiversity in Brazil. Rev. Bras. Bot. 30:587-597. 
MENDONÇA, M.P. \& LINS, L.V. 2000. Lista vermelha das espécies ameaçadas de extinção da flora de Minas Gerais. Fundação Biodiversitas; Fundação Zoo-Botânica de Belo Horizonte, Belo Horizonte.

MENDONÇA, R.C., FELFILI, J.M., WALTER, B.M.T., SILVA-Jr., M.C., REZENDE, A.V., FILGUEIRAS, T.S., NOGUEIRA, P.E. \& FAGG, C.W. 2008. Flora vascular do cerrado: Checklist com 12.356 espécies. In Cerrado: ecologia e flora (S.M. Sano, S.P. Almeida \& J.F. Ribeiro, ed.) EMBRAPA-CPAC, Planaltina, p.417-1279.

MIRANDA, S.C., SILVA-Jr., M.C. \& SALLES, L.A. 2007. A comunidade lenhosa de cerrado rupestre na Serra Dourada, Goiás. Heringeriana 1(1):43-53.

MOURA, I.O., GOMES-GLEIN, V.L., FELFILI, J.M. \& FERREIRA, H.D 2007. Fitossociologia da comunidade lenhosa de uma área de cerrado rupestre no Parque Estadual dos Pirineus, Pirenópolis, Goiás. Rev. Biol. Neotrop. 4(2):83-100.

MÜELLER-DOMBOIS, D. \& ELLENBERG, H. 1974. Aims and methods of vegetation ecology. J. Wiley \& Sons, New York.

MUNHOZ, C.B.R. \& PROENÇA, C. 1998. Composição florística no município de Alto Paraíso de Goiás na Chapada dos Veadeiros. Bol. Herb. Ezechias Paulo Heringer 3:102-150.

NOGUEIRA, E.M., NELSON, B.W., FEARNSIDE, P.M., FRANÇA, M.B. \& OLIVEIRA, A.C.A. 2008. Tree height in Brazil's "arc of deforestation": shorter trees in south and southwest Amazonia imply lower biomass. Forest Ecol. Manag. 255:2963-2972.

NOGUEIRA, P.E., FELFILI, J.M. \& SILVA-JÚNIOR, M.C. 2001 Composição florística e fitossociologia do cerrado sentido restrito no município de Canarana-MT. Bol. Herb. Ezechias Paulo Heringer 8:28-43.

PINTO, J.R.R., LENZA, E. \& PINTO, A.S. 2009. Composição florística e estrutura da vegetação arbustivo-arbórea em um cerrado rupestre, Cocalzinho de Goiás, Goiás. Rev. Bras. Bot. 32(1):23-32.
PRODEAGRO. 1995. Guia para identificação dos principais solos do Estado de Mato Grosso. SEPLAN/PRODEAGRO/PNUD, Cuiabá.

RABINOWITS, D. 1981. Seven forms of rarity, In The biological aspects of rare plant conservation (H. Synge, ed.) Jonh Wiley, Chicester, p.205-217.

RATTER, J.A., BRIDGEWATER, S. \& RIBEIRO, J.F. 2003. Analysis of the floristic composition of the Brazilian cerrado vegetation III: comparison of the woody vegetation of 376 areas. Edinb. J. Bot. 60(1):57-109.

RATTER, J.A., RIBEIRO, J.F. \& BRIDGEWATER, S. 1997. The Brazilian cerrado vegetation and threats to its biodiversity. Ann. Bot. 80:223-230.

REATTO, A., CORREIA, J.R., SPERA, S.T. \& MARTINS, E.S. 2008. Solos do bioma Cerrado: aspectos pedológicos. In Cerrado: ecologia e flora (S.M. Sano, S.P. Almeida \& J.F. Ribeiro, eds.). EMBRAPA-CPAC, Planaltina, p.107-150.

RIBEIRO, J.F. \& WALTER, B.M.T. 2008. Fitofisionomias do bioma Cerrado. In Cerrado: ecologia e flora (S.M. Sano, S.P. Almeida \& J.F. Ribeiro, eds.). EMBRAPA-CPAC, Planaltina, p.151-212.

ROHLF, F.J. 2000. NTSYS-pc: numerical taxonomy and multivariate analysis system, version 2.1. New York: Exeter Software.

SCOLFORO, J.R.S. 1993. Mensuração florestal 5: Crescimento florestal1. ESAL; FAEPE, Lavras.

SILVA, J.F., FARIÑAS, M.R., FELFILI, J.M. \& KLINK, C.A. 2006. Spatial heterogeneity, land use and conservation in the cerrado region of Brazil. J. Biogeogr. 33:536-548.

SILVA, L.O., COSTA, D.A., FILHO, K.E.S., FERREIRA, H.D. \& BRANDÃO, D. 2002. Levantamento florístico e fitossociológico em duas áreas de cerrado sensu stricto no Parque Estadual da Serra de Caldas Novas, Goiás. Acta Bot. Bras. 16(1):43-53.

SPIEGEL, M.P. 1976. Estatística. McGraw-Hill, São Paulo-SP. 